DOE/NV/25946--468

\title{
Reliable and Repeatable Characterization of Optical Streak Cameras
}

\author{
Michael R. Charest Jr.*, Peter Torres III*, Christopher T. Silbernagel*, Daniel H. Kalantar ${ }^{\dagger}$ \\ * National Security Technologies, LLC \\ 161-A S. Vasco Rd., Livermore, California, 94551 \\ Email: charesmr@nv.doe.gov \\ $\dagger$ Lawrence Livermore National Laboratory \\ 7000 East Ave., Livermore, California, 94551
}

\begin{abstract}
Optical streak cameras are used as primary diagnostics for a wide range of physics and laser experiments at facilities such as the National Ignition Facility (NIF). To meet the strict accuracy requirements needed for these experiments, the systematic nonlinearities of the streak cameras (attributed to nonlinearities in the optical and electrical components that make up the streak camera system) must be characterized. In some cases the characterization information is used as a guide to help determine how experiment data should be taken. In other cases, the characterization data are applied to the raw data images to correct for the nonlinearities. In order to characterize an optical streak camera, a specific set of data is collected, where the response to defined inputs are recorded. A set of analysis software routines has been developed to extract information such as spatial resolution, dynamic range, and temporal resolution from this data set. The routines are highly automated, requiring very little user input and thus provide very reliable and repeatable results that are not subject to interpretation. An emphasis on quality control has been placed on these routines due to the high importance of the camera characterization information.
\end{abstract}

\section{INTRODUCTION}

The characterization infrastructure described in this paper has been designed to meet several objectives: to extract the relevant calibration information in a standardized process while providing traceability. Traceability is a key element because incorrect characterization results may be propagated into experimental data. Retaining the full information about how the characterization was performed makes it possible to review data and identify issues associated with the calibration. Standardizing data collection and analysis allows direct comparisons between different streak camera systems as well as tracking of the performance of a single system over time. For our purposes here 'streak camera system' refers to the total of all the elements required to obtain a streaked image (including the temporal input slit, photocathode, and CCD readout). Part of standardizing both the data collection and the data analysis includes automating certain tasks. This has the added benefit of reducing human errors in the process as well as decreasing the time for a complete system characterization.

This paper provides a brief overview of the process we developed for characterizing optical streak cameras. We also highlight the analysis routines that were developed for characterizing streak camera flat field, dynamic range, spatial resolution, temporal resolution, and sweep linearity. Theses types of characterizations are fairly standard for streak cameras used in experimental setups. The novelty of this work lies in the standardization and automation of the data collection and analysis in our process. 


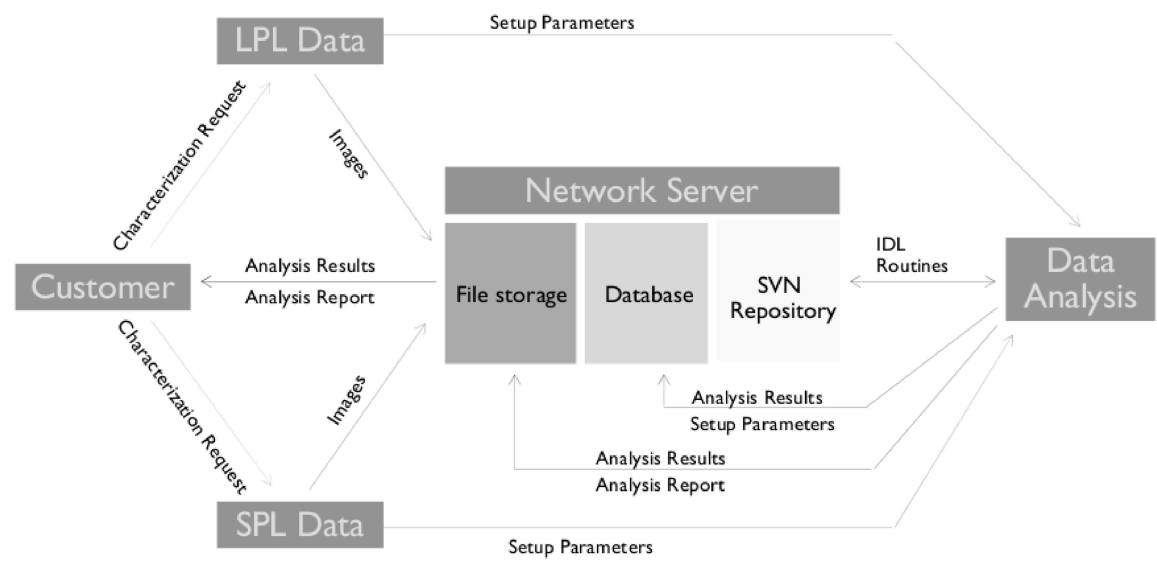

Fig. 1. Flow of data for an optical streak camera calibration.

\section{Characterization Data Flow}

The flow chart shown in Figure 1 describes, at a high level, how a streak camera characterization is carried out from start to finish. The process begins with a customer request that specifies the tests to be performed and the streak camera operating parameters for these tests (e.g. sweeps speeds, gain settings). This information is given to the laser operators. Data collection for optical streak camera characterization primarily takes place in two laser labs at the National Security Technologies (NSTec) Livermore Operations facility, the long pulse laser (LPL) laboratory and the short pulse laser (SPL) laboratory. The specifications for these lasers are shown in Figure 2.

\begin{tabular}{|c|c|c|c|}
\hline \multicolumn{4}{|c|}{$\begin{array}{l}\text { Long Pulse Laser Specifications } \\
\text { Nd:YAG Laser System (1064 nm Fundamental Wavelength) }\end{array}$} \\
\hline \multicolumn{2}{|c|}{ Pulse Energy Characteristics } & \multicolumn{2}{|c|}{ Temporal Pulse Characteristics } \\
\hline Wavelength & $\begin{array}{l}\text { Output Energy } \\
\text { per Pulse }\end{array}$ & \multirow{3}{*}{$\begin{array}{l}\text { Pulse Shape } \\
\text { Pulse Width } \\
\text { Rise Time }\end{array}$} & \multirow{2}{*}{$\begin{array}{l}\text { Flat Top Profile } \\
100 \mathrm{~ns}\end{array}$} \\
\hline $1064 \mathrm{~nm}$ & IJ & & \\
\hline $532 \mathrm{~nm}$ & $350 \mathrm{~mJ}$ & & $7 \mathrm{~ns}$ \\
\hline $355 \mathrm{~nm}$ & $100 \mathrm{~mJ}$ & Fall Time & $7 \mathrm{~ns}$ \\
\hline $266 \mathrm{~nm}$ & $200 \mathrm{~mJ}$ & \multirow[t]{2}{*}{ Repetition Rate } & \multirow[t]{2}{*}{$\mathrm{I} \mathrm{Hz}$} \\
\hline $213 \mathrm{~nm}$ & $80 \mathrm{~mJ}$ & & \\
\hline \multicolumn{4}{|c|}{ Short Pulse Laser Specifications } \\
\hline \multicolumn{4}{|c|}{ Ti:Sapphire Laser System ( $800 \mathrm{~nm}$ Fundamental Wavelength) } \\
\hline \multicolumn{2}{|c|}{ Pulse Energy Characteristics } & \multicolumn{2}{|c|}{ Temporal Pulse Characteristics } \\
\hline \multirow[t]{2}{*}{ Wavelength } & \multirow{2}{*}{$\begin{array}{l}\text { Output Energy } \\
\text { per Pulse }\end{array}$} & Pulse Shape & Impulse \\
\hline & & Pulse Width & $<1$ ps \\
\hline $400 \mathrm{~nm}$ & $2 \mathrm{~mJ}$ & Rise Time & - \\
\hline $266 \mathrm{~nm}$ & $500 \mu$ & Fall Time & $\cdot$ \\
\hline $200 \mathrm{~nm}$ & $150 \mu \mid$ & Repetition Rate & $10 \mathrm{~Hz}$ \\
\hline
\end{tabular}

Fig. 2. Laser facility specifications

The data are collected in the laser labs (following standardized procedures) and are stored on a network server. The data are then analyzed using the semi-automated analysis routines that are described in more detail later in this paper.

$\ddagger$ Contributed paper, published as part of the Proceedings of the 17th Topical Conference on High-Temperature Plasma Diagnostics, Albuquerque, New Mexico, May 2008.

* This work was done by National Security Technologies, LLC, under Contract No. DE-AC52-06NA25946 with the U.S. Department of Energy. DOE/NV/25946-468

$\dagger$ This work was done under the auspices of the U.S. Department of Energy by Lawrence Livermore National Laboratory under Contract DE-AC52-07NA27344. 


\section{A. Revision Traceability}

The data analysis routines that we have implemented are written in the Interactive Data Language (IDL [1]), an interpreted language distributed by ITT Visual Information Systems that is primarily geared toward data analysis and visualization applications. The routines are backed up on a network server in a Subversion (SVN [2]) repository. In the SVN repository, all versions of a file are tracked and retained, making it possible to easily revert to a previous version of a routine. We take advantage of this by additionally creating a tag within the repository (a snapshot of the current version of the routines) for each characterization analysis that we run. If a user needs to review the results of a particular characterization, quick and easy access is provided to the exact routine that was used.

\section{B. Analysis Reports}

Once an analysis routine has completed its analysis tasks, several other operations are performed. The analyzed results, the instrument settings, revision numbers, and other traceability information are saved to a Hierarchical Data Format (HDF [3]) archive and also to a database. A Portable Document Format (PDF) report is also generated. Several steps are involved in this creation of the report. First, figures that are generated as part of the calibration analysis are saved as encapsulated postscript files. The layout of the report is scripted using $\mathrm{I}_{\mathrm{E}} \mathrm{X}$, a typesetting language [4]. The $\mathrm{L}_{\mathrm{E}} \mathrm{X}$ file and encapsulated postscript images are then compiled into the final PDF format. The result is a high-quality, platform-independent document. The fact that the report is automatically generated is convenient for the analyst but also ensures that reports are consistent from characterization to characterization.

\section{ANALYSis Routines}

There are several different calibrations that must be applied to correct optical streak camera data. The main calibrations we perform are: flat field, dynamic range, spatial resolution, temporal resolution, and sweep linearity. Each of these calibrations is done at several sweep speeds as each sweep speed setting has its own unique characteristics.

\section{A. Flat Field}

The goal of the flat field characterization is to provide a correction image that can be applied to compensate for spatial and temporal sensitivity variations across the active area of the streak camera readout. The flat field characterization is performed by acquiring multiple swept images of a spatially and temporally flat illumination profile incident on the photocathode using the LPL. A total of ten images are used to create an average flat field image with reduced noise. This average image is then normalized and inverted to create a multiplicative image to correct for the non-uniform response of the camera. Additionally, regions of readout that are not illuminated by the streak camera are cropped using a threshold. Figure 3 shows a sequence of images, the first is an average of ten data images, the second is the multiplicative mask generated from the average image, and the third is the multiplicative mask after applying a threshold.
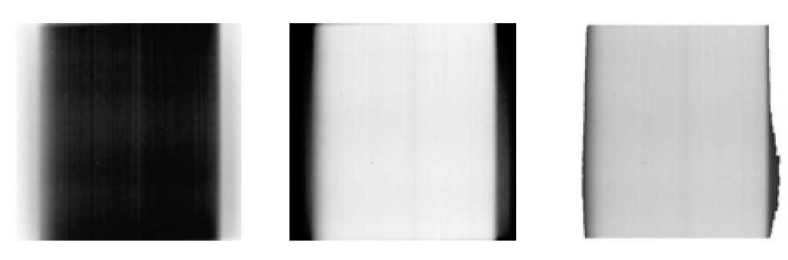

Fig. 3. This sequence shows the progression of the flat field correction mask at 3 different stages of the analysis. 


\section{B. Spatial Resolution}

The line-spread function (LSF) is a measurement of the streak camera spatial imaging resolution [5]. From the line spread function, one can obtain the contrast transfer function (CTF) as well. Both provide an indication of the streak cameras capability to resolve objects at various spatial frequencies. By imaging a multiple slit pattern mask, the LSF can be computed at many locations across the active area of the streak camera to generate a contour map of the spatial resolution (see Figure 4).

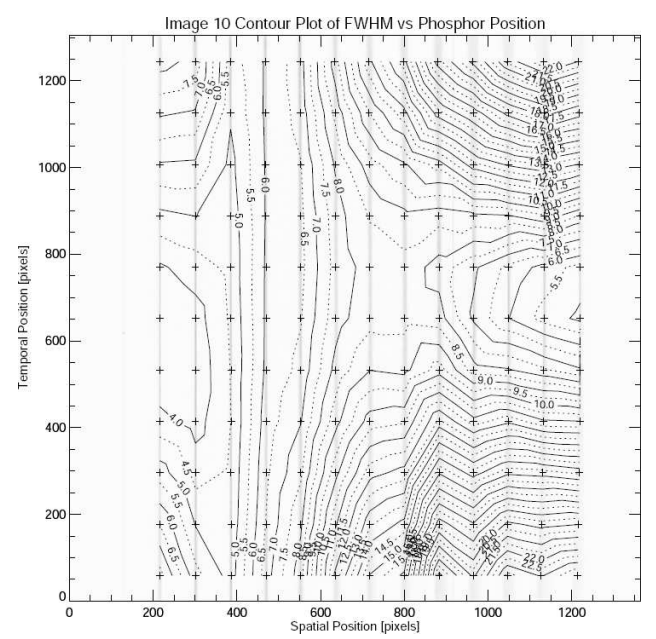

Fig. 4. The spatial resolution contour plot shows the FWHM of the LSF throughout the image.

This contour map can be used by an experimenter to optimize data collection to specific regions of the image in order to meet the spatial resolution requirements for the experiment. To obtain the LSF at any one location in the image, the profile of the slit image is fit with the convolution of an ideal rectangular function and a Gaussian function. The optimum Gaussian that fits the slit image profile is found using an iterative gradient descent method to provide the minimum least-squares solution. The spatial resolution is then quoted as the full-width-at-half-maximum (FWHM) of the best-fit Gaussian. This is then convolved with various spatial frequencies to determine the contrast transfer function.

\section{Temporal Resolution}

The temporal resolution is characterized using streaked images images of the extremely short duration SPL output. This allows a direct measurement of the temporal impulse response of the streak camera at specific locations. The trigger time for the streak camera is varied to acquire images at multiple positions on the screen (see Figure 5). A Gaussian Function is fit to the profile of the temporal slit at multiple spatial and temporal locations to generate a contour plot similar to the one created by the spatial resolution analysis.

\section{Dynamic Range}

The resolution of a streak camera degrades due to space-charge effects in the streak tube as the incident energy increases. Whether the temporal or spatial resolution degrades first depends on the streak camera system. To characterize the dynamic range based on spatial broadening, a series of images of a $50 \%$ illumination mask are taken with increasing input energy. A Gaussian curve is fit to the spatial profile of the center slit in each image and the FWHM of the curve is plotted vs. the average CCD count level of the image. A parabola is fit to this curve and the dynamic range is determined from the fitted curve. The upper limit of the dynamic range is defined as where the spatial resolution (fitted curve) increases by $20 \%$ over the minimum. Figure 6 is an example of a dynamic range plot. 

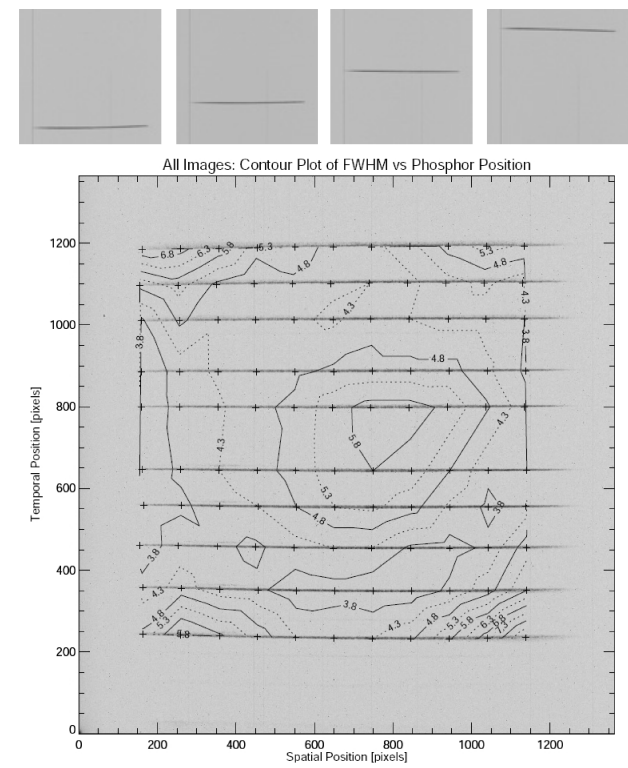

Fig. 5. Sequence of multiple temporal resolution images acquired with different trigger delays. These images are combined to derive the temporal resolution contour plot.

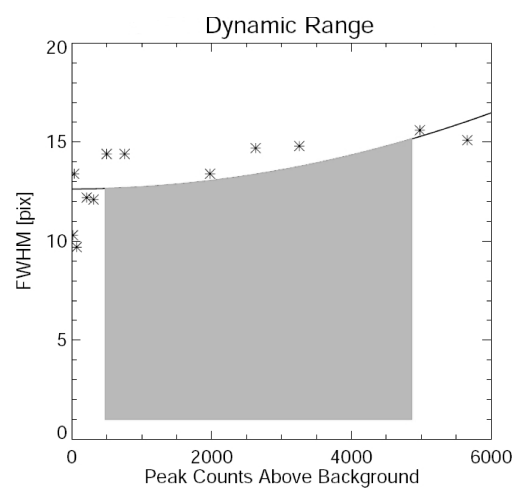

Fig. 6. This is an example to the dynamic range plot that indicates the linear response region of the streak camera.

The characterization of dynamic range based on temporal broadening is similar, except that the temporal slit of the streak camera is illuminated using the SPL. A Gaussian curve is fit to the temporal profile of the slit and the FWHM vs. CCD counts are plotted and fit following a method similar to that of the spatial broadening case.

\section{E. Sweep Linearity}

Using a known input of both space and time references, a streak camera image may be linearized in both space and time [6], [7]. The sweep linearity of a streak camera is characterized by using the same slit pattern mask used in the spatial resolution test. This pattern is illuminated by the SPL propagating through a fixed-space etalon, resulting in a linen-pattern image. A large number images are recorded, changing the trigger delay slightly between images to acquire a high density of pulses in the temporal direction of the sweep. The sweep linearity analysis routine locates the peak of each pulse in pixels in both space and time from images, and assigns absolute position in millimeters based on the slit position on the mask and relative time based on the etalon spacing. The relative timing of the different images is adjusted iteratively to develop a smooth sweep function consistent with the full dataset. The peak locations are defined as the from points for a geometric transformation. The go-to points are the true photocathode times and space positions of each pulse determined by the best fit between images. A geometric transformation matrix is determined using triangulation, and this may then be applied to raw data images in order to 
linearize them in both space and time. Additionally, by applying this transformation to an ideal ramp, an image representing the pixel dwell-time-image is generated. The dwell-time-image gives a visual representation of the sweep rate across the active area of the streak camera. Figure 7 shows examples of a linen-pattern image as well as a dwell-time-image.

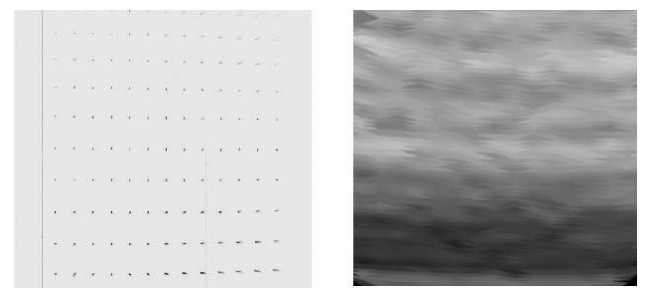

Fig. 7. This is an example of a linen pattern image used to generate the sweep linearity correction and an example dwell-time-image.

\section{SUMMARY}

A set of analysis routines has been designed to reliably produce accurate calibration results. Consistency and traceability are important to allow direct comparisons of streak camera systems as well as to allow tracking of the performance of individual streak camera systems over time. For quality assurance, the data storage system for calibration data is designed to allow quick access to the data needed to re-analyze a streak camera characterization or to answer any questions about precisely how the analysis results were attained. The reports that are produced as part of the analysis add a consistent mechanism for viewing summary analysis results. All of these elements combine to produce a highly repeatable and reliable characterization of a streak camera system. We have a similar process based on this model for characterizing other types of instruments such as gated imagers and CCD cameras. We are currently seeking accreditation from the National Institute of Standards and Technology (NIST [8]) through the National Voluntary Laboratory Accreditation Program (NVLAP), to help further the reliability of characterization processes. A large part of the accreditation process will be the inclusion of uncertainty as part of the analysis.

\section{REFERENCES}

[1] ITT Visual Information Systems. [Online]. Available: http://www.ittvis.com/

[2] Subversion. [Online]. Available: http://subversion.tigris.org/

[3] The HDF Group. [Online]. Available: http://hdf.ncsa.uiuc.edu/

[4] LATEX. [Online]. Available: http://www.latex-project.org/

[5] G. Boreman, Modulation Transfer Function in Optical and Electro-Optical Systems. SPIE Press, 2001.

[6] C. Silbernagel, P. T. III, and D. Kalantar, A method for analyzing high resolution, time domain, streak camera calibration data, in Advanced Signal Processing Algorithms, Architectures, and Implementations XIV, 2004, pp. 435442.

[7] D. Kalantar, P. Bell, R. Costa, A. Dymoke-Bradshaw, B. A. Hammel, J. Hares, O. Landen, and T. J. Orzechowski, Characterization of x-ray streak cameras for use on Nova, in 22nd International Congress on High Speed Photography and Photonics, 1997, pp. 680685.

[8] National Institute of Standards and Technology. [Online]. Available: http://www.nist.gov/ 